\title{
Reliability and Validity of the Dari version of the World Health Organization Quality of Life (WHOQOL-BREF) Questionnaire in Herat, Afghanistan: A cross-sectional study
}

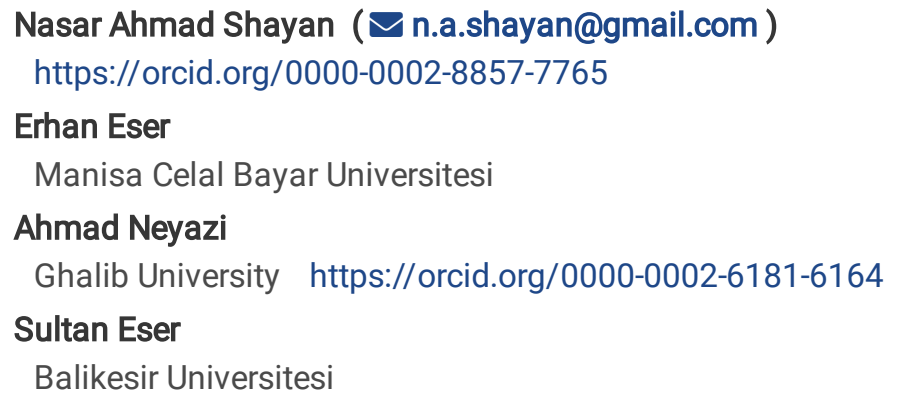




\section{Abstract \\ Background}

In order to predict social, mental and health care needs we must measure Quality of life of residents. In Afghanistan there was no assessment to measure quality of life of people. The aim of this study was to culturally adapt the WHOQOL-BREF into Dari language of Afghanistan and present its psychometric properties.

\section{Methods}

Cultural adaptation of the WHOQOL-BREF into Dari has consisted of two stages: translation stage, and the cognitive debriefing (pilot) stage following the translation stage. The process of translation of WHOQOL-BREF included recommended methodology, mandating forward translation, backward translation was followed. A total number of 1473 people did participate in this research. Psychometric analyses consisted of item analyses; reliability and validity analyses. Internal consistency analyses were done by Cronbach's alpha value and validity analyses consisted of construct validity (convergent validity by SF-36 and confirmatory factor analyses) and criterion validity (multiple linear regression by overall QoL item (item1)) analyses. Acceptable type 1 error was considered as 0.05 in all analyses. Analyses were done by using Lisrel v8.05 statistical package.

\section{Results}

Item domain correlations and -if item deleted-Cronbach alpha values no problematic item in Afghan version of the WHOQOL-BREF. Internal consistency of the scale was in acceptable limits for all domains (alpha values $=0.79-0.80$ ) except for the social relations domain (alpha $=0.41$ ). Using overall QoL item (item q1) as the dependent variable, multiple regression analysis revealed a R2 value of $57 \%$ and all of the four domains of the WHOQOL except that of Social relation domain (beta $=0.03$ ) could explain the variance of the $\mathrm{q} 1$, the best domain is the environmental domain with a beta value of 0.30 . Construct validity is tested by known groups validity; factorial validity and the convergent validity. All of the known categories of the age, gender, education, social class and economic status, having chronic illness were significantly sensitive to the domain scores of the WHOQOL $(p<0.001)$. Physical and Psychological domains of the WHOQOL-BREF was highly correlated with the related domains of the SF-36 $(r=0.60$ and 0.64$)$. Social and environmental domains showed poor correlations with the SF36. Confirmatory factor analyses showed acceptable goodness of fit results. Chi-square $=2174,748$ and Degrees of freedom $=246 ;$ GFI: 0.88, CFI: 0.83; RMR: 0.066 and RMSEA: 0.073 .

\section{Conclusion}

Afghan Dari version of the WHOQOL-BREF can confidently be used in clinical setting and in population level to assess the QoL of the people. The results of the social relations domain should be interpreted with caution due to its poor psychometric power. Further studies are needed to address the social aspects of quality of life in Afghan population.

\section{Introduction:}

Due to the technological development in medicine and increasing life expectancy in recent decades, beyond conventional health indicators, for example mortality and morbidity, there has been an increasing focus on measuring the quality of life (QoL) as a very important outcome in clinical settings as well as evaluations of the effects of different interventions on quality of life. World health organization (WHO) quality of life group defines the quality of life as "Individuals perception of their position in life in the context of the culture in which they live and the value systems they have about their goals, expectations, standards, and concerns" (1)(2)(3). Quality of life assessments have been widely used in health services in different purposes, either in health inequalities research or in clinical practice, to decide alternative treatment methods. Both generic and disease specific quality of life instruments have been developed to for different purposes in medical practice mostly used in combination with each other. Well developed, globally accepted and widely used generic quality of life instruments give the chance of international comparisons to the researchers. In earlier decades, a variety of instruments have been developed to measure the quality of life in different countries, though most of them are appropriate only in their relevant populations. However, some are culture-free and others are allowed to translate them into 
other languages for use in different societies after convenient development and localization. The world health organization (WHO) has developed a generic quality of life instrument which takes many subjective aspects of quality of life. The 100 item long (WHOQOL-100) and 26 item short (WHOQOL-BREF) versions of the WHOQOL that have been developed by the The WHOQOL group, serve as the official generic quality of life instruments of the WHO. The WHOQOL-100 covers six different domains and many of these domains seem to be cross-culturally important. It includes physical wellbeing, mental state, psychological state, social connections, individual's convictions and connections as salient features of the environment. Among the current QoL instruments available, the WHOQOL more likely to provide valid scores for comparison across community groups (4). The only problem with WHOQOL-100 is that, it is not easy to respond because of it is too long its lots of questions which makes the volunteer uncomfortable(5)(6)(7)(8)(9). The 26 item WHOQOL-BREF on the other hand, is one of the best among others and is available in more than 40 languages not only in normal populations but also recently in various diseases and conditions such as substance use disorder patients (10) and type two diabetes (11)(12)(13). It has only 26 questions as opposed to 100, though it aims to cover a broad range of quality of life facets divided into four main domains: The Physiological, Social, Physical and the Environmental. The WHOQOL-BREF has been translated and validated in many countries so far but has not been validated in Afghanistan yet (14)(15). A globally used, brief and cross culturally accepted generic quality of life tool is needed in health inequalities research and medical practice in Afghanistan. Dari is the first official language of Afghanistan, also known as Farsi or Afghan Persian.

The aim of this study is to culturally adopt the WHOQOL-BREF into Dari language of Afghanistan and test the reliability and validity of the Dari version of the WHOQOL-BREF.

\section{Methods:}

The WHOQOL-BREF is a 26 item brief version of the WHOQOL questionnaire. The WHOQOL-BREF covers four individual domains such as: Physical (activities of daily living, dependence on medicinal substances and medical aids, energy and fatigue, mobility, pain and discomfort, sleep and rest, work capacity); Physiological (bodily image and appearance, negative feelings, positive feelings, selfesteem, spirituality /religion/personal beliefs, thinking, learning, memory and concentration); Social (personal relationships, social support, sexual), and Environmental (financial resources, freedom/physical safety and security, health and social care: accessibility and quality, home environment, opportunities for acquiring new information and skills, participation in and opportunities for recreation/ leisure activities physical environment (pollution/ noise/ traffic / climate/ issues. Higher WHOQOL-BREF scale scores indicate better quality of life.

Afghanistan is a multi-ethnic country with Pashtuns, Tajiks, Uzbeks and Hazaras making up the majority of the population although Dari is one of the languages mostly used by the people of Herat city in Afghanistan and most of them are comfortable with Dari language.

\section{Linguistic And Cultural Adaptation:}

The cultural adaptation of the WHOQOL-BREF into Dari has consisted of two stages: translation stage, and the cognitive debriefing (pilot) stage following the translation stage. The process of translation of WHOQOL-BREF included recommended methodology, mandating forward translation, backward translation was followed. Forward translations were done by four independent translators and a consensus Dari version was developed by an expert linguist. The back translation of the Dari consensus version of the WHOQOL-BREF into English was done by a bilingual person. The back translated text was then compared with the original English version of the WHOQOL-BREF and a careful comparison was done between the two texts in terms of conceptual inconsistencies. When any inconsistency is detected between the two texts, the Dari version was revised accordingly, following a panel discussion with the translators.

Cognitive debriefing interviews were carried out on 30 healthy individuals. Following the completion of the questionnaire, each individual was asked to give their feedback about each of the items and response scales of the WHOQOL-BREF and each of the instruction sentences of the instrument. They were asked to tell:

" Whether they were able to understand each of the items and each of the instruction sentences or not?"

"If there is any word or phrase that they did not understand" if so, "how would they rephrase the question or the sentence?" 
They were also asked to do Thurston sort card exercise to confirm the correct order of response options in Dari version. It took almost 10 minutes for each individual to complete the questionnaire. The volunteers were able to understand all of the items and instructions with minimal modifications

\section{Study Sample And Data Collection:}

Field trial sample is a representative sample of Herat City, Afghanistan. The 2018 census of the Herat was around 270000 (16) The number of households is around 45000 in the city and the research was conducted on a household based stratified random sample of individuals aged 18 or above living in all 15 districts of Herat city. Volunteers consist of both healthy people (who stated that they are free of any kind of chronic disease) and ill participation (who stated that they have at least one or more medical condition receiving some form of medical care). Only one adult was selected from each of the households. 1473 persons were selected to the study sample (804 were males and 669 females). The gender imbalance was due to the higher willingness of men to participate to the study than women.

Of the participants, $53 \%$ were illiterate and $47 \%$ were literate. The inclusion criteria were being over age 18 and speaking the Dari language. Questionnaires were administered by an interviewer during face to face interviews in public.

\section{Psychometric Analyses:}

Psychometric analyses of the filed data of the WHOQOL-BREF Dari version is consisted of distribution properties and item analyses followed by internal consistency and validity analyses. Minimal acceptable limits for Floor and Ceiling value percentages are $\leq 20 \%$ (17) and Skewness and Kurtosis limits are 2.0 (18).

Both reliability and Validity analysis were based on confirmatory approach. Internal consistency of the individual domains were tested by Cronbach's alpha (19) The average inter-item correlations for domains, and correlation of items with their intended domain were also calculated to assess scale success statistics. If Pearson's product moment coefficients between the items and their own scale score (corrected for overlap) were higher than the correlations between these items and any other domain then its regarded that WHOQOL domains represent separate entities. "If item removed alpha values" and item scale correlations were used to detect any problematic items. If any domain's alpha value increases when internal consistency analyses in repeated when a certain item removed, then that item might be a problematic item. The problematic item may be proved by looking its correlation coefficient with its own dimension score if it is less than 0.30 .

Convergent-divergent validity, known groups' validity and confirmatory factor analysis were employed for the assessment of construct validity of the WHOQOL-BREF Dari version. Sf-36 was used to test convergent-divergent validity of the WHOQOL-BREF and gender, education, social class and presence of any illness were used for known groups'/discriminant analyses. The discriminative ability of the instrument between a subgroups were assessed by Student's $t$ test indicating the known groups validity of the measurement. Effect size statistics (i.e. mean differences divided by pooled sd.) were computed to determine the magnitude of the difference in mean scores (20). A Cohen's D (ES) value closer to 0.20 indicates a small effect, whereas 0.50 a medium and 0.8 and over a big effect in two groups' comparisons. A third approach that was used in this manuscript for testing the construct validity of the WHOQOL-BREF-DARI was the Confirmatory Factor Analyses (CFA), type of Structural Equation Modelling. Fit indices generated by CFA were used to test the original WHOQOL scale structure of the Dari version of the WHOQOL-BREF. Root Mean Square Error Approximation (RMSEA) and Comparative Fit Index (CFI) Tucker Levis Index (TLI) and Root Mean Residuals (RMR) and Chi square tests. Critical acceptable threshold is 0.90 for CFI and TLI and 0.08 for RMSEA and RMR (18).

The construct validity of the measurement is examined by LISREL 8.5. Criterion validity was tested by linear regression analysis, taking general quality of item (q1) as the criterion of quality of life. Type 1 error is taken as 0.05 in all statistical analyses.

\section{Results}

\section{Socio-demographic characteristics}

Page $4 / 11$ 
Of the 1473 respondents, $54.6 \%$ men and $45.4 \%$ women. The $38.0 \%$ of the study sample was $18-29$ years old, while $14.8 \%$ was $30-$ $39 ; 24.6 \%$ was $40-49$ years old and $22.6 \%$ was 50 and over, with a mean age of $37.6 \pm 14.2$ years ( $\min 14, \max 86)$. Only $47.25 \%$ (n $=696)$ of the respondents were graduated from any school $; 41.96 \%(n=618)$ were illiterate and $10.79 \%(n=159)$ were just know how to read and write. $25.4 \%$ of the respondents perceived themselves as poor; $46.0 \%$ as moderate and $28.6 \%$ as good or wealthy. About one quarter of the respondents self-reported an existence of an important illness (25.2\%). Only $6.5 \%$ was current smokers and a great majority of the study sample (89.1\%) stated that they had no access to health services.

\section{Psychometric Results}

Item frequency analyses showed that the floor and ceiling effects range for each domains are in acceptable limits for all domains. The range of the floor effects was 0.0 to $0.3 \%$ and ceiling effects was 0.1 to $3.2 \%$ for the domains of WHOQOL-BREF. Skewness and Kurtosis values showed that all of the four domain scores were normally distributed $(<1.0)($ Table 1$)$.

Table 1

Item descriptive statistics and reliability properties (Cronbach's alphas, correlation coefficients)

\begin{tabular}{|c|c|c|c|c|c|c|c|c|}
\hline $\begin{array}{l}\text { Dimensions of the } \\
\text { WHOQOL-BREF }\end{array}$ & $\begin{array}{l}\text { Mean } \pm \\
\text { SD\# }\end{array}$ & $\begin{array}{l}\text { Floor } \\
\%\end{array}$ & $\begin{array}{l}\text { Ceiling } \\
\%\end{array}$ & $\begin{array}{l}\text { Skewness } \\
\text { (SE) }\end{array}$ & $\begin{array}{l}\text { Kurtosis } \\
\text { (SE) }\end{array}$ & $\begin{array}{l}\text { a (if item deleted } \\
\text { alpha range) }\end{array}$ & $\begin{array}{l}\text { Cor. } \\
\text { rage } \\
\text { (r) }\end{array}$ & $\begin{array}{l}\text { Item Success } \\
\text { Rate \% }\end{array}$ \\
\hline Physical health & $\begin{array}{l}62,6 \pm \\
17.2\end{array}$ & 0.0 & 0.5 &,$- 32(.06)$ & $\begin{array}{l}-, 11 \\
(.13)\end{array}$ & $0.79(0.74-0.78)$ & $\begin{array}{l}0.63- \\
0.75\end{array}$ & 100 \\
\hline Psychological health & $\begin{array}{l}60.5 \pm \\
17.8\end{array}$ & 0.0 & 0.6 &,$- 17(.06)$ &,$- 42(.13)$ & $0.79(0.73-0.78)$ & $\begin{array}{l}0.62- \\
0.79\end{array}$ & 100 \\
\hline Social Relations & $\begin{array}{l}62.1 \pm \\
19.4\end{array}$ & 0.3 & 3.2 &,$- 30(.06)$ &,$- 11(.13)$ & $0.41(0.22-0.37)$ & $\begin{array}{l}0.65- \\
0.75\end{array}$ & 100 \\
\hline Environmental health & $\begin{array}{l}52.0 \pm \\
16.4\end{array}$ & 0.2 & 0.1 &,$- 06(.06)$ & ,24(.13) & $0.80(0.77-0.79)$ & $\begin{array}{l}0.60- \\
0.70\end{array}$ & 100 \\
\hline \multicolumn{9}{|c|}{ a: Cronbach's alpha value; Cor. range: Range of Item-scale correlation coefficients (corrected for overlap); } \\
\hline
\end{tabular}

The Cronbach's alpha values of all of the domains were satisfactory ( 0.79 to 0.80$)$ except for the social relations domain which is quite low (0.41). Item-scale and "if item removed alpha" values indicated no problematic items of the WHOQOL-DARI version. All of the 26 items were correlated with their own domains higher than with other domains, indicating 100\% "item success". (Table 1).

Construct validity is tested by convergent validity, known groups validity and factorial validity approaches. Physical and Psychological domain scores of the WHOQOL- BREF and SF-36 are highly correlated each other (good convergence) ( $r=0.52$ and 0.60 ), whereas environmental domain of the WHOQOL is not correlated with neither physical nor psychological sub dimensions of the SF-36 (poor convergence) as expected. Social domain of the WHOQOL fails to be correlated with the social dimension of the SF36 indicating poor convergence. (Table 2). 
Table 2

Correlation between the dimensions of the WHOQOL-BREF and SF-26 scales for convergent-discriminant validity

\begin{tabular}{|c|c|c|c|c|c|c|c|c|}
\hline Dimensions & SF-36 & & & & & & & \\
\hline \multirow[t]{2}{*}{$\begin{array}{l}\text { WHOQOL- } \\
\text { BREF }\end{array}$} & $\begin{array}{l}\text { Physical } \\
\text { function }\end{array}$ & $\begin{array}{l}\text { Role } \\
\text { limit. }\end{array}$ & Pain & $\begin{array}{l}\text { General } \\
\text { health }\end{array}$ & Energy & $\begin{array}{l}\text { Social } \\
\text { function }\end{array}$ & $\begin{array}{l}\text { Role limit. } \\
\text { Emotion }\end{array}$ & $\begin{array}{l}\text { Emotion. Well- } \\
\text { being }\end{array}$ \\
\hline & & $\begin{array}{l}\text { Physical } \\
\text { health }\end{array}$ & & & & & & \\
\hline $\begin{array}{l}\text { Physical } \\
\text { health }\end{array}$ &, $517(* \star)$ &, $375(* \star)$ &, $580(* *)$ &, $638(* *)$ &, $599(* *)$ &, $506(* *)$ &, $336(* \star)$ &, $548(* *)$ \\
\hline $\begin{array}{l}\text { Psychological } \\
\text { health }\end{array}$ &, $332(* *)$ &, $266(* *)$ &, $\left.4400^{\star *}\right)$ &, $580(* *)$ &, $585(\star \star)$ &, $473(* \star)$ &, $305\left(^{\star *}\right)$ &, $602\left(^{\star *}\right)$ \\
\hline $\begin{array}{l}\text { Social } \\
\text { Relations }\end{array}$ &, $162(* *)$ &, $133\left(^{\star *}\right)$ &, $\left.194{ }^{\star * *}\right)$ &, $309\left(^{\star *}\right)$ &, $320(* *)$ &, $\left.230{ }^{* \star}\right)$ &, $126(* \star)$ &, $320(* \star)$ \\
\hline $\begin{array}{l}\text { Environmental } \\
\text { health }\end{array}$ &, $100(* *)$ &, $114(* \star)$ &, $185\left(^{\star *}\right)$ &, $304(* \star)$ &, $292(* *)$ &, $202\left(^{\star *}\right)$ &, $122(* \star)$ &, $284(* \star)$ \\
\hline$\star * x<0.01$ & & & & & & & & \\
\hline
\end{tabular}

As for the know groups validity analyses, all of the known categories of the gender, level of education, social class and having chronic illness were significantly sensitive to all of the four domain scores of the WHOQOL $(p<0.001)$. We see that women were much worse than men in terms of physical, psychological and environmental dimensions, whereas social class has moderate to high level of discriminable effect on all of the four domains. The physical well-being domain score was more negatively affected by having a chronic illness compared to other domains. Environmental health domain was more sensitive to socioeconomic independent variables such as level of education and social class and BMI was only sensitive to physical domain. (Table 3 ).

Table 3

Known groups validity: Effect size differences in WHOQOL-BREF

\begin{tabular}{|c|c|c|c|c|c|}
\hline & Gender & Education & Social Class & illness & BMI 『 \\
\hline & $\begin{array}{l}\text { Female }< \\
\text { Male }\end{array}$ & $\begin{array}{l}\text { Illiterate }<\text { (Literate only = } \\
\text { Educated) }\end{array}$ & $\begin{array}{l}\text { Lower }<\text { middle }< \\
\text { upper }\end{array}$ & $\begin{array}{l}\text { ill< } \\
\text { well }\end{array}$ & $30.0<-29.99$ \\
\hline Physical health & $0.56 * \star \star$ & 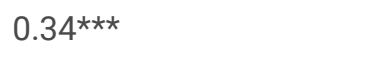 & 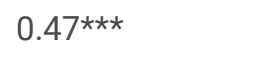 & $1.23^{\star \star \star}$ & $0.31^{\star \star}$ \\
\hline Psychological health & 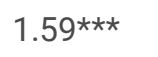 & $0.39 * * *$ & $0.63 * * *$ & $0.43 * \star \star$ & 0.25 \\
\hline Social Relations & $0.30 * \star \star$ & $0.32^{\star *}$ & $0.41 * * \star$ & $0.39 * \star \star$ & 0.08 \\
\hline $\begin{array}{l}\text { Environmental } \\
\text { health }\end{array}$ & $1.09 * * *$ & $0.78 * * *$ & $0.75 * \star \star$ & $0.27 * \star$ & $0.36 *$ \\
\hline \multicolumn{6}{|c|}{ Effect size differences in WHOQOL-BREF dimension scored by socio-demographic and physical health conditions. } \\
\hline \multicolumn{6}{|c|}{ Effect Size (Cohen's d): two groups: 0.2 low, 0.5 medium, 0.8 large; three or more groups: 0.2 low, 0.5 medium, 0.8 large; } \\
\hline \multicolumn{6}{|c|}{$\star p<0.05, * * p<0.01, * * \star p<0.001$} \\
\hline \multicolumn{6}{|c|}{$\nabla$ Body Mass Index categories: $<18.5=1 ; 18.66$ to $24.99=2 ; 25.00$ to $29.99=3 ; 30.00$ and over $=4$} \\
\hline
\end{tabular}

Confirmatory factor analyses showed acceptable goodness of fit results for RMR (0.066) and RMSEA (0.073), but GFI (0.88) and CFI (0.83) values were lower than expected. (Table 4). 
Table 4

Summary reports of

confirmatory factor

analyses of the

WHOQOL-BREF Afghan

Version

\begin{tabular}{|ll|}
\hline Fit indices & Value \\
\hline RMSEA & 0.073 \\
\hline CFI & 0.83 \\
\hline GFI & 0.88 \\
\hline TLI & 0.81 \\
\hline Stand.RMR & 0.066 \\
\hline X2 & 2174 \\
\hline Df & 246 \\
\hline
\end{tabular}

Criterion validity was tested by multiple regression analysis using overall QoL item (item q1) as the dependent variable. The regression model revealed a R2 value of $57 \%$. All of the four domains of the WHOQOL-BREF-Dari except that of Social relation domain (beta $=0.03$ ) could explain the variance of the Q1. The best predicting domain is environmental domain on overall QoL. Social relations domain could not predict overall QoL at al. (Table 5).

Table 5

Criterion validity of the WHOQOL-BREF

\begin{tabular}{|lrrrrr|}
\hline & \multicolumn{5}{c}{ Collinearity Statistics } \\
\hline Dependent variable (overall quality of life item) & Beta & $\mathrm{p}$ & VIF & Std. Error \\
\hline Constant & &, 007 & & \\
\hline Psychological health & 0.10 &, 001 & 0.51 & 1,97 \\
\hline Social Relations & 0.28 &, 000 & 0.45 & 2,30 \\
\hline Environmental health & 0.03 &, 161 & 0.74 & 1,36 \\
\hline Physical health & 0.30 &, 000 & 0.73 & 1,37 \\
\hline (Linear Regression model: The Overall QoL item as dependent variable; R2 = 0.35) \\
\hline
\end{tabular}

\section{Discussion:}

Recent political developments in Afghanistan are expected to have positive influences on the health sector of the country that covers broader approach to determinants of health and the assessment of the outcomes of the health interventions both in public health and in the clinical practice. As one of the specific aspects of the assessment of the well-being of the population quality of life assessments need to be done in addition to objective health measures in Afghanistan. This study assessed the cultural relevance and psychometric properties of the Afghan Dari language version of the WHOQOL-Bref.

Linguistic adaptation of the original WHOQOL-Bref to Dari language followed the strict international translation procedures including multi forward and backward translation and cognitive interviews in the Herat city, the third biggest city of the country. The study sample is a representative large sample, allowing the generalizability of the results to Dari -the leading official language of the country- speaking community of Afghanistan.

The distribution properties of the WHOQOL-Bref-Dari were found quite satisfactory with very low floor and ceiling effects and acceptable Skewness and Kurtosis value limits, consistent with the results of several studies in the literature (8)(11)(21)(22)(23)(24). Internal consistency of the scale was assessed by Cronbach Alpha value and three of four dimensions of the WHOQOL-Dari revealed 
adequate alpha values ( 0.79 to 0.80 ) except for Social Relations dimension which had an alpha value of 0.41 . Similar lower alpha values of Social Relations dimension were obtained in some other studies conducted in Bangladesh, Denmark, UK and Norway (1) (25)(26)(27) A possible explanation of this low internal consistency in social relations dimension is the misconception of the items by the illiterate respondents. A stratified analyses of illiteracy/literacy (not presented in the results) showed that the alpha value is 0.38 in illiterate people whereas it is 0.43 for literate people. Additionally, we felt the necessity of exploring existence of any problematic item and we run "if item deleted alpha values" for each of the dimensions and found no problematic item in any of the dimensions of WHOQOL-Dari, including the social relation dimension. What makes us some comfortable is the moderate to high item-dimension correlations $(0.60-0.79)$ for all dimensions including the social relations dimension of the WHOQOL-Dari.

We tested the construct validity of the WHOQOL-Dari by three different methods: Convergent-divergent validity, known groups validity and the factor analyses. The generic SF-36 scale was used to assess the convergent validity of the WHOQOL-Bref-Dari version. Physical health dimension score of the WHOQOL revealed higher correlation coefficients with the physical health related dimension scores of the SF-36 than the emotional dimension scores of the SF-36. Also a moderate convergence was found between WHOQOL psychological dimension score and the Emotional Well-being dimension score of the SF-36. Social relations dimension and the Environmental dimension scores of the WHOQOL, had poorer convergence with the related dimension of the SF-36. Our results are consistent with some other papers in that, the Environmental health dimension of the WHOQOL had no significant correlations with any of the dimensions of the SF-36 (27). The poor convergence of the WHOQOL Environmental dimension with neither physical nor mental dimension scores may be due its content of socio-economic drive. Hence Environmental health dimension represents the socioeconomic well-being of the respondent and was not sensitive to any health related concepts which was shown in several studies including original WHOQOL-group papers (6) (24) (28)(29) .

Lower Environmental dimension scores were also obtained from the less developed European study sites such as Romania and Turkey in the WHOQOL global study mentioned above. Our known groups validity results also confirmed the higher sensitivity of the environmental health score to the socioeconomic indicators: Quite big effect size figures were obtained for education (0.78) and social class (0.75) variables. On the other hand, several studies indicated that there is not a good convergence between WHOQOL and SF-36 (29) (30) (31).

The Known groups validity analyzes are performed to show previously proven relationships. The women, the ill and low educated people and also those people who belonged to a lower social class are previously known as disadvantaged groups in the community in regard to quality of life. Our results indicated that, all four dimensions of the WHOQOL Bref-Dari version are sensitive to gender, education, social class, objective health status, consistent with literature findings (5) (6)(7) 29).

The confirmatory factor analyses results, as the third method of testing the construct validity of the WHOQOL-Dari version showed moderate to high goodness of fit results. RMSEA index and RMR were both in acceptable limits $(<0.08)$ whereas confirmatory fit indices were around 0.83 to 0.88 showing moderate fit to the original WHOQOL-Bref scale structure. In contrary with the satisfactory RMR and RMSEA findings, moderate CFI results were obtained in several other WHOQOL validation studies (32) (33).

Criterion validity of the WHOQOL-Dari version is shown by a linear regression of the Overall QoL item of the WHOQOL over the domain scores. The best Beta values were obtained for Physical health and Social relations dimensions. The only non-significant dimension is the environmental dimension. This is consistent with the finding that environmental dimension is the weakest related dimension with illness variable, that was shown in the known groups validity findings of our study.

There are several limitations of this study. First, the WHOQOL-Bref has been face to face administered to the respondents due to the high portion (about $42 \%$ ) of illiterate population, which might positively bias the results of this validation study. Secondly, it was not always possible to fulfill personal privacy during the interviews. And finally the men/women participation rate was higher in favor of men due to the social restrictions of the Afghan community.

To conclude we can say that, WHOQOL-Bref-Dari version can be confidently used in public health programs and clinical practice in Dari speaking communities of Afghanistan, but Social relations dimension scores should be interpreted with caution.

\section{Conclusion}


As we know, this is the first study on Afghanistan's general population demonstrating the relation between socio-demographic variables and QoL domains. Afghan Dari version of the WHOQOL-BREF can confidently be used in clinical setting and in population level to assess the QoL of the people. The results of the social relations domain should be interpreted with caution due to its poor psychometric power. Further studies are needed to address the social aspects of quality of life in Afghan population.

\section{Abbreviations}

WHOQOL-Bref: World Health Organization Quality of Life- Bref; QoL:Quality of Life; RMSEA:The Root Mean Square Error of Approximation; CFI:Confirmatory factor analyses; SF-36:Short form survey 36; GFI:comparative fit index; RMR:Root Mean Square Residual; TLI:Tucker-Lewis index; Df:degrees of freedom; $\chi 2$ :chi square

\section{Declarations}

\section{Acknowledgement}

Not applicable.

\section{Competing interests}

The authors declare that they have no competing interests.

\section{Funding}

This research was funded by Ghalib University, Faculty of Medicine, Herat, Afghanistan.

\section{Authors' contributions}

- NAS and AN did contribute in study concept, design and acquisition of data, analysis, interoperation and data collection.

- ER and SE contributed to data analysis.

- NAS and AN wrote manuscript draft.

- ER and SE revised and finalized the manuscript.

- All the authors approved the final revision for publishing.

\section{Ethics approval}

The study was ethically approved by Ghalib University. A detailed description of the study, benefits, confidentiality and the informed consent procedures was explained during the initial contact with prospective participants prior to their participation. All the collected information from this study would be treated confidentially and only accessible by the members of the research.

\section{Consent for publication}

A written informed consent was gained for all participants.

\section{References}

1. Noerholm V, Groenvold M, Watt T, Bjorner JB, Rasmussen NA, Bech P. Quality of life in the Danish general population-normative data and validity of WHOQOL-BREF using Rasch and item response theory models. Qual Life Res. 2004 Mar;13(2):531-40.

2. Cheung YB, Yeo KK, Chong KJ, Khoo EYH, Wee HL. Measurement equivalence of the English, Chinese and Malay versions of the World Health Organization quality of life (WHOQOL-BREF) questionnaires. Health and Quality of Life Outcomes. 2019 Apr 17;17(1):67.

3. Lin C-Y, Lee T-Y, Sun Z-J, Yang Y-C, Wu J-S, Ou H-T. Development of diabetes-specific quality of life module to be in conjunction with the World Health Organization quality of life scale brief version (WHOQOL-BREF). Health Qual Life Outcomes. 2017 Aug 23;15(1):167.

4. A systematic and critical review of the process of translation and adaptation of generic health-related quality of life measures in 
Bowden A, Fox-Rushby JA. A systematic and critical review of the process of translation and adaptation of generic healthrelated quality of life measures in Africa, Asia, Eastern Europe, the Middle East, South America. Soc Sci Med. 2003;57(7):1289306.

5. Sreedevi A, Cherkil S, Kuttikattu DS, Kamalamma L, Oldenburg B. Validation of WHOQOL-BREF in Malayalam and Determinants of Quality of Life Among People With Type 2 Diabetes in Kerala, India. Asia Pac J Public Health. 2016 Jan;28(1 Suppl):62S69S.

6. Lodhi FS, Montazeri A, Nedjat S, Mahmoodi M, Farooq U, Yaseri M, et al. Assessing the quality of life among Pakistani general population and their associated factors by using the World Health Organization's quality of life instrument (WHOQOL-BREF): a population based cross-sectional study. Health Qual Life Outcomes. 2019 Jan 14;17(1):9.

7. Skevington SM, Lotfy M, O'Connell KA, WHOQOL Group. The World Health Organization's WHOQOL-BREF quality of life assessment: psychometric properties and results of the international field trial. A report from the WHOQOL group. Qual Life Res. 2004 Mar;13(2):299-310.

8. Bonomi AE, Patrick DL, Bushnell DM, Martin M. Validation of the United States' version of the World Health Organization Quality of Life (WHOQOL) instrument. J Clin Epidemiol. 2000 Jan;53(1):1-12.

9. Dalky HF, Meininger JC, Al-Ali NM. The Reliability and Validity of the Arabic World Health Organization Quality of Life-BREF Instrument Among Family Caregivers of Relatives With Psychiatric Illnesses in Jordan. J Nurs Res. 2017 Jun;25(3):224-30.

10. Muller AE, Skurtveit S, Clausen T. Performance of the WHOQOL-BREF among Norwegian substance use disorder patients. BMC Medical Research Methodology. 2019 Mar 4;19(1):44.

11. Colbourn T, Masache G, Skordis-Worrall J. Development, reliability and validity of the Chichewa WHOQOL-BREF in adults in Lilongwe, Malawi. BMC Res Notes. 2012 Jul;3:5:346.

12. Nedjat S, Montazeri A, Holakouie K, Mohammad K, Majdzadeh R. Psychometric properties of the Iranian interview-administered version of the World Health Organization's Quality of Life Questionnaire (WHOQOL-BREF): A population-based study. BMC Health Services Research. 2008 Mar;21(1):61. 8(.

13. Reba K, Birhane BW, Gutema H. Validity and Reliability of the Amharic Version of the World Health Organization's Quality of Life Questionnaire (WHOQOL-BREF) in Patients with Diagnosed Type 2 Diabetes in Felege Hiwot Referral Hospital, Ethiopia [Internet]. Vol. 2019, Journal of Diabetes Research. Hindawi; 2019 [cited 2020 May 6]. p. e3513159. Available from: https://www.hindawi.com/journals/jdr/2019/3513159/.

14. Yao G, Chung C-W, Yu C-F, Wang J-D. Development and verification of validity and reliability of the WHOQOL-BREF Taiwan version. J Formos Med Assoc. 2002 May;101(5):342-51.

15. The Whoqol Group. The World Health Organization quality of life assessment (WHOQOL): Development and general psychometric properties. Social Science \& Medicine. 1998 Jun 15;46(12):1569-85.

16. World Population Review. Population of Cities in Afghanistan. (2020) [Internet]. 2020 World Population by Country. [cited 2020 May 9]. Available from: https://worldpopulationreview.com/countries/afghanistan-population/cities/ (accessed 20 march 2020).

17. Andresen EM. Criteria for assessing the tools of disability outcomes research. Arch Phys Med Rehabil. 2000 Dec;81(1):15-20.

18. Hooper D, Coughlan J, Mullen M. Structural Equation Modelling: Guidelines for Determining Model Fit. Articles [Internet]. 2008 Jan 1; Available from: https://arrow.tudublin.ie/buschmanart/2.

19. Nunnally JC. Psychometric Theory 3E. Tata McGraw-Hill Education; 1994. 784 p.

20. Cohen J. Statistical Power Analysis. Curr Dir Psychol Sci. 1992 Jun 1;1(3):98-101.

21. Krägeloh CU, Kersten P, Rex Billington D, Hsu PH-C, Shepherd D, Landon J, et al. Validation of the WHOQOL-BREF quality of life questionnaire for general use in New Zealand: confirmatory factor analysis and Rasch analysis. Qual Life Res. 2013 Aug;22(6):1451-7.

22. Jaracz K, Kalfoss M, Górna K, Baczyk G. Quality of life in Polish respondents: psychometric properties of the Polish WHOQOLBref. Scand J Caring Sci. 2006 Sep;20(3):251-60.

23. Suárez L, Tay B, Abdullah F. Psychometric properties of the World Health Organization WHOQOL-BREF Quality of Life assessment in Singapore. Qual Life Res. 2018 Nov;27(11):2945-52. 
24. Redko C, Rogers N, Bule L, Siad H, Choh A. Development and validation of the Somali WHOQOL-BREF among refugees living in the USA. Qual Life Res. 2015 Jun;24(6):1503-13.

25. Izutsu T, Tsutsumi A, Islam A, Matsuo Y, Yamada HS, Kurita H, et al. Validity and reliability of the Bangla version of WHOQOLBREF on an adolescent population in Bangladesh. Qual Life Res. 2005 Sep;14(7):1783-9.

26. Yuan-Tao HAO, Ji-Qian HFANG, Power F. MJ P. The Equivalence of WHOQOL-BREF among 13 Culture Versions. Chinese Mental Health Journal [Internet]. 2006 [cited 2020 May 18]; Available from: http://en.cnki.com.cn/Article_en/CJFDTotalZXWS200602000.htm.

27. A T, T I, Ma SK, Hs I Y, H K, et al. Reliability and Validity of the Bangla Version of WHOQOL-BREF in an Adult Population in Dhaka, Bangladesh [Internet]. Vol. 60, Psychiatry and clinical neurosciences. Psychiatry Clin Neurosci; 2006 [cited 2020 May 20]. Available from: https://pubmed.ncbi.nlm.nih.gov/16884453/.

28. Skevington SM, Lotfy M, O'Connell KA, WHOQOL Group. The World Health Organization's WHOQOL-BREF quality of life assessment: psychometric properties and results of the international field trial. A report from the WHOQOL group. Qual Life Res. 2004 Mar;13(2):299-310.

29. Eser E, Fidaner H, Fidaner C, Elbi H, Göker E. Psychometric properties of the WHOQOL-100 and WHOQOL-BREF. 3P Dergisi. Jan. 1999;1:7:23-40.

30. Castro PC, Driusso P, Oishi J. Convergent validity between SF-36 and WHOQOL-BREF in older adults. Rev Saude Publica. 2014 Feb;48(1):63-7.

31. Chang C-Y, Huang C-K, Chang Y-Y, Tai C-M, Lin J-T, Wang J-D. Cross-validation of the Taiwan version of the Moorehead-Ardelt Quality of Life Questionnaire II with WHOQOL and SF-36. Obesity surgery. 2009 Apr 1;20:1568-74.

32. CW GY, Cf C, Jd Y W. Development and verification of validity and reliability of the WHOQOL-BREF Taiwan version. J Formos Med Assoc. 2002 May 1;101(5):342-51.

33. Silva WR da, Bonafé FSS, Marôco J, Maloa BFS, Campos JADB. Psychometric properties of the World Health Organization Quality of Life Instrument-Abbreviated version in Portuguese-speaking adults from three different countries. Trends Psychiatry Psychother. 2018;40(2):104-13. 